\title{
The Adverse Effects of Antidepressant Medication Treatments on the Offspring of Women with Perinatal Depression
}

\author{
Amira Mohammed Ali ${ }^{1 *}$, Afaf Ahmed Hassan ${ }^{2}$ and Amin Omar Hendawy ${ }^{3,4}$ \\ ${ }^{1}$ Department of Psychiatric Nursing and Mental Health, Alexandria University, Egypt \\ ${ }^{2}$ Department of Obstetrics and Gynecological Nursing, Alexandria University, Egypt \\ ${ }^{3}$ Department of Animal and Poultry Production, Damanhour University, Egypt \\ ${ }^{4}$ Department of Biological Production, Tokyo University of Agriculture and Technology, Japan
}

*Corresponding author: Amira Mohammed Ali, Department of Psychiatric Nursing and Mental Health Alexandria university, Egypt.

Received Date: October 20, 2018

Published Date: January 09, 2019

\begin{abstract}
Background: Antidepressants are considered the first line of treatment of depression, which prevails during pregnancy and following child birth.

Objective: This paper aims to review, synthesize, and discuss the available literature regarding the effect of using antidepressants during pregnancy/lactation on pregnancy outcomes as well as fetal and neonatal status. Whenever possible, we elaborate on the mechanisms behind these effects.

Results: Use of antidepressants during pregnancy is associated with birth complications such as premature labor. These drugs cross the placenta and access the fetal circulation and organs, which is associated with congenital anomalies and impairment of motor and neurodevelopment during childhood. Antidepressants vary according their level of excretion in human milk; however, the literature on the effect of these drugs on nursing babies is controversial.

Conclusion: Antidepressants use during pregnancy represents a threat to the growing fetus; whenever possible safer alternatives should be considered. Further rigorous investigations of the effect of these drugs on breastfed babies are necessary.
\end{abstract}

Keywords: Antidepressant medications; Adverse effects; Congenital anomalies; Perinatal depression; Pregnancy; Lactation

Abbreviations: IGF2: Insulin-like Growth Factor 2; NGF: Nerve Growth Factor; OXTR: Oxytocin Receptor Gene; SERT: Serotonin Transporter; SLC6A4: Fetal Serotonin Transporter Gene; SNRIs: Serotonin Norepinephrine Reuptake Inhibitors; SSRIs: Selective Serotonin Reuptake Inhibitors; TeCA: Tetracyclic Antidepressant.

\section{Introduction}

Up to one fifth of pregnant and postpartum women are affected by depressive disorders, which imply a range of adverse consequences for both moms, their babies, and the entire family $[1,2]$. Untreated prenatal maternal depression is reported to induce epigenetic fetal changes that involve permanent alteration of the neuroendocrine functions of the fetus. As a result of such a stressed fetal neurodevelopment, these children are at a great risk of delayed cognitive and motor development as well as impaired tolerance to stressful conditions $[3,4]$. In the same way, postnatal depression represents a risk for non-optimal parenting behaviors, impaired mother-child interactions, child victimization, and development of childhood psychopathology [5,6].

Antidepressant medications-and in particular, selective serotonin reuptake inhibitors (SSRIs)-are considered as the first line of treatment of perinatal depression $[7,8]$. It is estimated that $10 \%$ of pregnant women are prescribed antidepressant medications [8]. To achieve treatment goals, these drugs are consumed for long periods of time (at least 6-8 months) since treatment of depression 
involves acute phase management to achieve remission and maintenance phase, which aims to prevent recurrence of the same episode or future episodes $[9,10]$. In other words, not only moms but also their fetus/babies will be exposed to antidepressants for a long time. Effective treatment standards should ensure that benefits of treatment of depression outweigh its drawbacks. In fact, the therapeutic effectiveness of antidepressants during pregnancy and lactation is not well-established. A recent meta-analytic review indicated that the effect of SSRIs during the perinatal period does not outperform the efficacy of other active treatments such as cognitive behavioral therapies-the latter are expensive and time consuming [11]. In line, a recent Cochrane review could not locate any evidence of effectiveness of antidepressants for preventing postnatal depression. Some of the participants who received these drugs developed manic and hypomanic episodes, while side effects such as constipation, dizziness, and drowsiness were more common among women taking antidepressants compared with those who received placebo treatments [12].

Concerning the safety profile of these drugs during pregnancy and lactation, several systematic reviews indicate that the safety of antidepressants during pregnancy is not well-known $[13,14]$, while another review indicates that intake of SSRIs represent a harmful chemical exposure to mothers and their fetus [15]. It is noteworthy that prolonged use of antidepressant medications (even at therapeutic doses) may contribute to oxidative stress, immunomodulation, inflammation, and homeaostatic imbalance, which are pathological mechanisms of depression and several other serious disorders e.g., cardiotoxicity, diabetes, cancer, and obesity [16-20]. Interestingly, a recent review reported that antidepressants excreted in human wastes decreased reproduction, increased mortality; and caused developmental retardation, morphological anomalies, and pathological changes in the brain, heart, cranial and caudal kidney of several aquatic creatures [21]. These findings are quite alarming. The use of antidepressants during pregnancy and lactation may represent a potential source of teratogenic risks for the mother as well as the growing fetus. Herein, we explore evidence concerning the various effects that antidepressants could have on pregnancy outcomes and fetus/neonates biochemicals. Whenever possible, we elaborate on the mechanisms behind these effects.

\section{The effect of antidepressants on pregnancy outcomes and fetus/neonates}

Maternal exposure to antidepressants during pregnancy may negatively affect birth outcomes. Former reviews report that antenatal use of SSRIs and other antidepressants such as mirtazapine is associated with an increased risk of spontaneous abortion and preterm birth $[10,14,22]$. Alike, several review studies document a significant association between antenatal use of antidepressants and perinatal complications such as lower rates of live births and higher rates of newborns with low birth weight $[2,10,13,23]$. Another review indicates that all mood stabilizing drugs are associated with risks of birth defects-problems related to intrauterine development of the fetus, especially during the first three months of pregnancy [2]. In addition, antenatal intake of antidepressants (e.g., SSRIs and mirtazapine) is associated with increased risks of fetal tachyarrhythmia [4], persistent neonatal pulmonary hypertension, poor neonatal adaptation syndrome, hypoglycemia, and transient neonatal symptoms in up to one-third of newborns $[14,22,24]$.

On the other hand, mixed findings are reported on the association of antidepressant use during pregnancy with congenital malformations. Two reviews report that major congenital malformations are less likely to be associated with prenatal use of Serotonin Norepinephrine Reuptake Inhibitors (SNRIs) and mirtazapine-a tetracyclic antidepressant (TeCA) [13,14]. On the other hand, a considerable number of systematic reviews report that maternal exposure to SSRIs such as escitalopram and paroxetine during pregnancy (especially during the first trimester) affects the developing fetus and increases the risk of various congenital malformations such as congenital cardiac anomalies [2,22-24]. A former study reported that the risk of cardiovascular malformations associated with paroxetine (expressed as adjusted odds ratio) increased from 1.46 (95\%CI $0.74-2.88$ ) for monotherapy to 1.68 (95\%CI 0.95-2.97) for combination with other antidepressants [25].

\section{The effect of gestational exposure to antidepressants on growing children}

Evidence indicates that antidepressants used during pregnancy cross the placenta, and they are constantly accessible to the fetal circulation via amniotic fluid [26,27]. An animal model reported that both fluoxetine and norfluoxetine, an active metabolite of fluoxetine, cross the placenta and enter tissues of the brain, kidney, and other organs of the fetus [28]. Such exposure may represent unfavorable intrauterine environmental condition that is associated with suboptimal fetal growth [10] and long-lasting epigenetic modifications that can ultimately affect normal development and increase susceptibility to chronic diseases in later life $[4,29]$. The literature suggests that embryo exposure to antidepressants is associated with both motor and cognitive alterations during childhood. A recent meta-analysis and a former review reported a significant association of gestational exposure to antidepressants with delayed motor development and overall occurrence of poor motor outcomes in childhood (effect size $=0.22$; $95 \%$ confidence interval $=0.07$ to 0.37$)[22,30]$. It is noteworthy that children with significant motor problems experience a variety of negative psychosocial consequences such as poorer attention and learning ability, lower self-esteem and self-efficacy, lower perceived social support, and higher levels of emotional distress than matched controls [30].

Furthermore, the literature suggests that children of women who receive antidepressants during pregnancy are at a high risk of neurodevelopment impairment. Perinatal exposure to SSRIs is reported to negatively affect the plasticity of developing hippocampus especially in male fetus, which affects children's behavioral development [31]. In accordance, a number of recent systematic reviews and meta-analyses report that antenatal use of antidepressants (SSRIs) increases the risk of attention-deficit/ hyperactivity disorder and autism spectrum disorders [7,32-34]. 


\section{The effect of exposure of nursing infants to antidepressant contents of breast milk}

Mothers are recommended to exclusively breastfeed their babies especially during the first year of life since breast feeding is a source of enormous benefits for mothers and their babies [35]. However, antidepressants can be excreted in breast milk and transferred to the newborn [26]. A former study reported that the maternal oral dose of tricyclic antidepressants was significantly associated with the amounts of these drugs and their metabolites in maternal plasma and in breast milk [36]. In line, a meta-analysis study assessed the association between infant plasma levels of antidepressants with maternal levels of antidepressants both in plasma and milk. That study indicated that the excretion of antidepressants in breast milk and its effect on the plasma of the baby differ from one drug to another: nortriptyline, paroxetine, and sertraline had undetectable infant levels, whereas fluoxetine had the highest proportion (22\%) of infant levels-that were 10\% higher than the average maternal level. Levels of citalopram were elevated in $17 \%$ of infants [37]. Another meta-analysis reported that levels of sertraline and its principle metabolite (desmethylsertraline) were $87.4 \%$ and $70 \%$ below the limit of detection in infants breastfed by mothers who received that drug. However, a significant correlation was revealed between maternal and infant desmethylsertraline concentrations [38]. Results concerning the effect of antidepressants content of breast milk on nursing infants are mixed and scattered. An earlier review reported that the safety profiles of sertraline and paroxetine during lactation are better relative to other SSRIs; however, the literature is deficient on studies that investigate the safety profile of most antidepressants. In the meantime, rigorous studies that evaluate the long-term neurodevelopmental outcomes in infants breastfed by women on antidepressants are lacking [39]. A previous study reported that the daily doses of antidepressants ingested by breastfed babies constituted $1 \%$ of the maternal dose/ $\mathrm{kg}$, and no acute toxicity or developmental impairments were noted in ten breastfed infants [36]. In another study, the antidepressant breast milk-to-plasma ratio ranged between $51.1 \%$ and $73.4 \%$. No neonatal side effects were indicated-however, a large percentage of women (42\%) discontinued breast feeding one week after birth [40]. Similarly, a recent prospective study that followed 280 infants whose mothers received psychotropic monotherapy during lactation reported no adverse effects of these drugs on children's growth and gross motor development. However, infant sleepiness was highly reported [35]. Another report, on the contrary, indicated that maternal use of amitriptyline at a dose of $10 \mathrm{mg} /$ day was reported to cause severe neonatal sedation and poor sucking [41].

\section{Discussion}

This minireview aimed to shed light on the adverse effects exhibited by exposure of embryos and nursing infants to maternal antidepressant medications. A considerable number of reviews and meta-analyses confirm that fetal exposure to antidepressants is associated with a range of fetal problems such as stunted fetal growth. A trail of motor impairments and cognitive problems such as autism ensue in children born for women who received antidepressants during pregnancy. One possible mechanism that explains how maternal exposure to antidepressants affects the fetus is the contribution of these drugs to placenta DNA methylation of the oxytocin receptor gene (OXTR), which plays a major role in the alteration of maternal-fetal-placental stress physiology. OXTR DNA methylation severely alters the fetal stresssensitive biological systems [6]. In particular, DNA methylation of fetal serotonin transporter gene (SLC6A4) in the human placenta is sensitive to the maternal metabolic state in pregnancy, and it was positively associated with maternal blood glucose level [42]. Antidepressant drugs function by elevating levels of biogenic amines, serotonin in particular [43] Serotonin is a multifunctional neurotransmitter that plays a major role in glucose regulation. The serotonin transporter (SERT, 5HTT) is a basic protein of the neuron membrane. 5HT homeostasis is a key function of this transporter, while SERT employs a high-affinity transport mechanism to regulate 5HT uptake. Most antidepressants target the function of serotonin transporter [42]. Antagonism of 5-HT2C receptors and M3 muscarinic receptors reduces the secretion of insulin from pancreatic beta cells. It is documented that antidepressants induce several metabolic disturbances by contributing to insulin sensitivity, glucose intolerance, hyperlipidemia and the like $[44,45]$. This mechanism seems to be supported by findings of a number of studies. A former study explored the effect fetal exposure to maternal depression or antidepressants on OXTR DNA methylation at two differentially methylated regions of the imprinted Insulinlike Growth Factor 2 (IGF2) gene. This study reported no effect of depression on methylation profiles. However, intrauterine exposure to antidepressants was associated with a significant hypermethylation of the H19 DMRs in newborns of African American-not Caucasian-mothers $(\beta=+6.89, \mathrm{p}=0.01)$, which is considered a race-dependent response to antidepressants [29]. Similarly, a recent study reported that antidepressant use during pregnancy was associated with placenta DNA methylation even after adjustment for depressive symptoms. Cord plasma antidepressant levels were strongly associated with increased DNA methylation greater than its association with maternal antidepressant dose or blood level of antidepressants [30]. In accordance, a third study reported that both SSRIs treatment and untreated antenatal depression negatively affect placental development and function by altering the expression of placental genes: six out of seven of 20 selected genes were altered in SSRI treated women compared with controls (healthy pregnant women), and two genes were altered between depressed women and controls. Similarly, both conditions altered the expression of placental proteins that are involved in the nerve growth factor (NGF) signaling pathway: NGF, phosphorylated Raf-1, ROCK2 and phosphorylated ROCK2. Such placental alterations could ultimately affect fetal development [46]. Likewise, a recent review reported that antidepressants and untreated maternal depression negatively affect placental functioning and induce epigenetic changes that interfere with the physiological behavior and the biochemical architecture of the growing fetus [4].

Another mechanism involves the effect of direct embryo exposure to antidepressants on the neurodevelopment of the growing fetus. The therapeutic action of SSRIs involves increasing serotonin levels in the synaptic cleft through binding to SERT (the 
serotonin transporter) and blocking reabsorption of serotonin by presynaptic neurons [26]. Serotonin is a multifunctional signaling molecule that appears very early in fetal development. It regulates various brain development processes in the human brain: neurogenesis, migration, differentiation, and other aspects of brain morphogenesis. More, it auto-regulates the development of its own serotonergic neurons and aids in the development of neurons which produce other neurotransmitters [26,30,42]. Given that SSRIs readily cross the placenta and the fetal blood-brain barrierwhich is not mature till after birth; there is a high probability of perturbations to the neurobiology, alteration of serotonin signaling, and development of serotonin circuitry, which ultimately increase the risk of neurodevelopmental disorders in exposed embryos $[8,30]$. Disturbances of serotonin homeostasis play a major role in the pathogenesis of several disorders: depression, autism and other mental health conditions as well as obesity and related metabolic disorders [42]. In support of this mechanism, a former study reported that human embryos that were treated with fluoxetine exhibited alterations of embryo developmental stages. More, levels of proteins secreted from fluoxetine-treated embryo were altered compared with untreated embryos [46].

Overall, the literature on the effect of use of antidepressants on the breastfed infants and whether these drugs should be used during lactation is controversial. A former review reports that serious acute adverse effects from drugs in breast milk are rare. Nonetheless, special attention is necessary in very young infants (under the age of 2 months) since they are most susceptible to effects of drugs that target the central nervous system [47]. Another review recommends that mothers should carefully monitor their newborns for adverse effects (irritability, poor feeding, or uneasy sleep, especially if the child was born premature or had low birth weight). The lowest effective dose should be considered, and whenever possible, breastfeeding should be avoided at the time when the milk concentration of antidepressants is at its peak [48].

\section{Conclusion}

Overall, it seems that maternal use of antidepressants during pregnancy represents a threat to the growing fetus and might negatively affect motor and cognitive development after birth. Whenever possible safer alternatives should be considered. The literature on the effect of postpartum maternal use of antidepressants on nursing infants is deficient. Further rigorous investigations of the effect of these drugs on breastfed babies are necessary.

\section{Acknowledgement}

None.

\section{Conflict of Interest}

The authors have no conflict of interest to declare.

\section{References}

1. Glynn LM, Davis EP, Sandman CA (2013) New insights into the role of perinatal HPA-axis dysregulation in postpartum depression. Neuropeptides. 47(6): 363-370.

2. Vitale SG, Lagana AS, Muscatello MR, La Rosa VL, Curro V, et al. (2016) Psychopharmacotherapy in Pregnancy and Breastfeeding. Obstet Gynecol Surv. 71(12): 721-733.
3. Fatima M, Srivastav S, Mondal AC (2017) Prenatal stress and depression associated neuronal development in neonates. Int J Dev Neuroscience. 60: 1-7.

4. Gentile S, Fusco ML (2017) Placental and fetal effects of antenatal exposure to antidepressants or untreated maternal depression. The Journal of Maternal-Fetal \& Neonatal Medicine 30(10): 1189-1199.

5. Plant DT, Jones FW, Pariante CM, Pawlby S (2017) Association between maternal childhood trauma and offspring childhood psychopathology: Mediation analysis from the ALSPAC cohort. British Journal of Psychiatry 211(3): 144-150.

6. Toepfer P, Heim C, Entringer S, Binder E, Wadhwa P, et al. (2017) Oxytocin pathways in the intergenerational transmission of maternal early life stress. Neuroscience \& Biobehavioral Reviews 73: 293-308.

7. Fatima Z, Zahra A, Ghouse M, Yang L, Wang X, et al (2018) Maternal SSRIs Experience and Risk of ASD in Offspring's: A Review. Toxicology Research.

8. Millard SJ, Weston-Green K, Newell KA (2017) The effects of maternal antidepressant use on offspring behavior and brain development: Implications for risk of neurodevelopmental disorders 80: 743-765.

9. Biffi A (2017) Antidepressants and the risk of cardiovascular diseases. (Doctorate), Università Degli Studi di Milano-Bicocca.

10. Zhao X, Liu Q Cao S, Pang J, Zhang H, et al. (2018) A meta-analysis of selective serotonin reuptake inhibitors (SSRIs) use during prenatal depression and risk of low birth weight and small for gestational age. 241: 563-570.

11. Nillni YI, Mehralizade A, Mayer L, Milanovic S (2018) Treatment of depression, anxiety and trauma-related disorders during the perinatal period: A systematic review. 66: 136-148.

12. Molyneaux E, Telesia LA, Henshaw C, Boath E, Bradley E, et al. (2018) Antidepressants for preventing postnatal depression. Cochrane Database Syst Rev 4.

13. Bellantuono C, Vargas M, Mandarelli G, Nardi B, Martini MG (2015) The safety of serotonin-noradrenaline reuptake inhibitors (SNRIs) in pregnancy and breastfeeding: a comprehensive review. Hum Psychopharmacology 30(3): 143-151.

14. Smit M, Dolman KM, Honig A (2016) Mirtazapine in pregnancy and lactation - A systematic review. Eur Neuropsychopharmacology 26(1): 126-135.

15. Urato AC (2015) Are the SSRI antidepressants safe in pregnancy? Understanding the debate. Int J Risk Saf Med 27(2): 93-99.

16. Ali AM, Hendawy AO (2018) Bee Honey as a Potentially Effective Treatment for Depression: A Review of Clinical and Preclinical Findings. JOJ Nurse Health Care, 9(2).

17. Andersohn F, Schade R, Suissa S, Garbe E (2009) Long-term use of antidepressants for depressive disorders and the risk of diabetes mellitus. Am J Psychiatry 166(5): 591-598.

18. Bennett MI (2010) Effectiveness of antiepileptic or antidepressant drugs when added to opioids for cancer pain: systematic review. Palliative Medicine. 25(5): 553-559.

19. Holt RI, De Groot M, Golden SH (2014) Diabetes and depression. Curr Diab Rep 14(6): 491.

20. Taylor D (2008) Antidepressant drugs and cardiovascular pathology: a clinical overview of effectiveness and safety. Acta Psychiatr Scand 118(6): 434-442.

21. Sehonova P, Svobodova Z, Dolezelova P, Vosmerova P, Faggio C (2018) Effects of waterborne antidepressants on non-target animals living in the aquatic environment: A review. Sci Total Environ 1: 631-632: 789794.

22. Pearlstein T (2015) Depression during Pregnancy. Best Pract Res Clin Obstet Gynaecol 29(5): 754-764.

23. Bellantuono C, Orsolini L, Bozzi F (2013) The safety profile of escitalopram in pregnancy and breastfeeding. Rive Psychiatry 48(6): 407-414. 
24. Balkowiec-Iskra E, Mirowska-Guzel DM, Wielgos M (2017) Effect of antidepressants use in pregnancy on fetus development and adverse effects in newborns. Ginekol Pol 88(1): 36-42.

25. Cole JA, Ephross SA, Cosmatos IS, Walker AM (2007) Paroxetine in the first trimester and the prevalence of congenital malformations. Pharmacoepidemiology and Drug Safety 16(10): 1075-1085.

26. Lattimore KA, Donn SM, Kaciroti N, Kemper AR, Neal CR, et al. (2005) Selective serotonin reuptake inhibitor (SSRI) use during pregnancy and effects on the fetus and newborn: a meta-analysis. J Perinatol. 25(9): 595-604.

27. Paulzen M, Goecke TW, Stickeler E, Gründer G, Schoretsanitis G (2017) Sertraline in pregnancy - Therapeutic drug monitoring in maternal blood, amniotic fluid and cord blood. J Affect Disord 212: 1-6.

28. Pohland RC, Byrd TK, Hamilton M, Koons JR (1989) Placental transfer and fetal distribution of fluoxetine in the rat. Toxicology and Applied Pharmacology 98(2): 198-205.

29. Soubry A, Murphy SK, Huang Z, Murtha A, Schildkraut JM, et al. (2011) The effects of depression and use of antidepressive medicines during pregnancy on the methylation status of the IGF2 imprinted control regions in the offspring. 3(1): 2 .

30. Grove K, Lewis AJ, Galbally M (2018) Prenatal Antidepressant Exposure and Child Motor Development: A Meta-analysis. Pediatrics, 142(1).

31. Pawluski JL, Gemmel M (2018) Perinatal SSRI medications and offspring hippocampal plasticity: interaction with maternal stress and sex. 17(1): $15-24$.

32. Man KKC, Chan EW, Ip P, Coghill D, Simonoff E, et al. (2018) Prenatal antidepressant exposure and the risk of attention-deficit hyperactivity disorder in children: A systematic review and meta-analysis. 86: 1-11.

33. Morales DR, Slattery J, Evans S, Kurz X (2018) Antidepressant use during pregnancy and risk of autism spectrum disorder and attention deficit hyperactivity disorder: systematic review of observational studies and methodological considerations. BMC Medicine. 16(1): 6.

34. Uguz F (2018) Maternal Antidepressant Use During Pregnancy and the Risk of Attention-Deficit/Hyperactivity Disorder in Children: A Systematic Review of the Current Literature. J Clin Psychopharmacology 38(3): 254-259.

35. Kronenfeld N, Ziv Baran T, Berlin M, Karra N, Dinavitser N, et al. (2018) Chronic use of psychotropic medications in breastfeeding women: Is it safe? PLoS One, 13(5).

36. Yoshida K, Smith B, Craggs M, Channi Kumar R (1997) Investigation of pharmacokinetics and of possible adverse effects in infants exposed to tricyclic antidepressants in breast-milk. J Affect Disord 43(3): 225-237.
37. Alicia M Weissman, Barcey T Levy, Arthur J Hartz, Suzanne Bentler, Micca Donohue, et al. (2004) Pooled Analysis of Antidepressant Levels in Lactating Mothers, Breast Milk, and Nursing Infants. American Journal of Psychiatry 161(6): 1066-1078.

38. Pinheiro E, Bogen DL, Hoxha D, Ciolino JD, Wisner KL (2015) Sertraline and breastfeeding: review and meta-analysis. Arch Womens Ment Health. 18(2): 139-146.

39. Orsolini L, Bellantuono C (2015) Serotonin reuptake inhibitors and breastfeeding: a systematic review. Hum Psychopharmacology 30(1): 4-20.

40. Pogliani L, Baldelli S, Cattaneo D, Pileri P, Clementi E, et al. (2018) Selective serotonin reuptake inhibitors' passage into human milk of lactating women. The Journal of Maternal-Fetal \& Neonatal Medicine 6: $1-6$.

41. Faruk U (2017) Poor Feeding and Severe Sedation in a Newborn Nursed by a Mother on a Low Dose of Amitriptyline. Breastfeeding Medicine 12(1): 67-68.

42. Blazevic S, Horvaticek M, Kesic M, Zill P, Hranilovic D, et al. (2017) Epigenetic adaptation of the placental serotonin transporter gene (SLC6A4) to gestational diabetes mellitus. 12(6).

43. Leuchter AF, Hunter AM, Krantz DE, Cook IA (2015) Rhythms and blues: modulation of oscillatory synchrony and the mechanism of action of antidepressant treatments. Annals of the New York Academy of Sciences. 1344(1): 78-91.

44. Abosi O, Lopes S, Schmitz S, Fiedorowicz JG (2018) Cardiometabolic effects of psychotropic medications. Horm Mol Biol Clin Investing.

45. Tran YH, Schuiling-Veninga CCM, Bergman JEH, Groen H, Wilffert B (2017) Impact of Muscarinic M3 Receptor Antagonism on the Risk of Type 2 Diabetes in Antidepressant-Treated Patients: A Case-Controlled Study. CNS Drugs 31(6): 483-493.

46. Kaihola H (2015) The effects of SSRI treatment on human placenta and embryo (Doctorate Doctor of Philosophy), Uppsala University, Digital Comprehensive Summaries of Uppsala Dissertations from the Faculty of Medicine 1095.

47. Anderson PO, Manoguerra AS, Valdes V (2016) A Review of Adverse Reactions in Infants from Medications in Breastmilk. Clin Pediatric (Phila) 55(3): 236-244.

48. Cuomo A, Maina G, Neal SM, De Montis G, Rosso G, et al. (2018) Using sertraline in postpartum and breastfeeding: balancing risks and benefits. Expert Opin Drug Safe 17(7): 719-725. 